\title{
Effects of Cholestasis on Whole Blood Concentration of Tacrolimus, an Immunosuppressant, in Living-Related Liver Transplant Recipients"
}

\author{
Shinji Kobuchi ${ }^{1 \#}$, Keizo Fukushima ${ }^{2}$, Yuta Maeda ${ }^{1}$, Takatoshi Kokuhu ${ }^{3}$, Hidetaka Ushigome ${ }^{4}$, \\ Norio Yoshimura ${ }^{4}$, Nobuyuki Sugioka ${ }^{2}$, Kanji Takada ${ }^{1}$ \\ ${ }^{1}$ Department of Pharmacokinetics, Kyoto Pharmaceutical University, Kyoto, Japan; ${ }^{2}$ Department of Clinical Pharmacokinetics, Fac- \\ ulty of Pharmaceutical Sciences, Kobe Gakuin University, Kobe, Japan; ${ }^{3}$ Department of Hospital Pharmacy, Kyoto Prefectural Uni- \\ versity of Medicine, Kyoto, Japan; ${ }^{4}$ Department of Transplantation and Regenerative Surgery, Kyoto Prefectural University of Medi- \\ cine, Kyoto, Japan. \\ Email: "ky05122@poppy.kyoto-phu.ac.jp
}

Received July $19^{\text {th }}, 2013$; revised August $20^{\text {th }}, 2013$; accepted September $15^{\text {th }}, 2013$

Copyright (C) 2013 Shinji Kobuchi et al. This is an open access article distributed under the Creative Commons Attribution License, which permits unrestricted use, distribution, and reproduction in any medium, provided the original work is properly cited.

\begin{abstract}
Background: To explore the effects of cholestasis on whole blood concentration of tacrolimus (TAC), an immunosuppressant, we investigated the relationship among blood TAC concentration, bile flow, and TAC metabolites in bile, as well as the relationship between total bilirubin (T-Bil), an index of cholestasis, and blood TAC concentration, in liver transplant recipients. Methods: Data were collected retrospectively from 16 male and 19 female patients (mean age: 38 years; range: 12 - 59 years) who had undergone a living-related liver transplantation at Kyoto Prefectural University of Medicine from 2004 through 2008. Analysis of TAC, demethyl-TAC, and hydroxy-TAC in bile was performed by LC-MS/MS. Results: There was no correlation between the ratio of TAC metabolite to TAC in bile (M/P) of demethyl-TAC and post operation days (POD), whereas a weak linear correlation was demonstrated between $\mathrm{M} / \mathrm{P}$ of hydroxy-TAC and POD $(r=-0.345)$. Moreover, linear correlations were not observed between M/P and the TAC trough level normalized dose $\left(\mathrm{TL}_{\mathrm{TAC}} /\right.$ dose), and between $\mathrm{TL}_{\mathrm{TAC}} /$ dose and POD. A negative linear correlation was demonstrated between bile flow and T-Bil in blood $(r=-0.495)$. Furthermore, a positive linear correlation was observed between $\mathrm{TL}_{\mathrm{TAC}} /$ dose and T-Bil $(r=0.598)$, whereas there was no correlation between bile flow and $\mathrm{TL}_{\mathrm{TAC}} /$ dose. Conclusions: Improvement of hepatic function and the increase of TAC clearance after postoperative day 7 did not significantly contribute to hepatic $\mathrm{TAC}$ metabolism, bile excretion, and $\mathrm{TL}_{\mathrm{TAC}}$ /dose. Postoperative biliary stricture from liver transplantation with/without biliary drainage caused inter- and intra-patient variability in $\mathrm{TL}_{\mathrm{TAC}}$ /dose after liver transplantation, which could be assessed by T-Bil. T-Bil in blood might be a predictive biomarker for determining the degree of bile duct stricture and TAC dose in liver transplantation patients. Along with an appropriate dosing regimen, therapeutic drug monitoring including T-Bil would be beneficial and enable individual adjustment of TAC dose in liver transplantation patients.
\end{abstract}

Keywords: Pharmacokinetics; Bile Duct Stricture; Tacrolimus; Drug Metabolism; Total Bilirubin; Therapeutic Drug Monitoring

\section{Introduction}

Tacrolimus (TAC), which is an immunosuppressive agent (molecular weight of non-hydrate: 806), inhibits the signal-transduction pathway that leads to T-lymphocyte activation $[1,2]$. TAC is used for preventing or treating graft rejection after organ transplantation [3],

\footnotetext{
*The authors declare that no conflict of interest exists.

${ }^{\#}$ Corresponding author.
}

and is superior to cyclosporine (CyA) in improving survival (patient and graft) and preventing acute rejection in living-related liver transplantation [4]. TAC is used as the first choice drug for living-related liver transplant recipients at our university hospital, Kyoto Prefectural University of Medicine. Pharmacokinetic parameters of TAC show high variability, particularly in the early period after transplantation, which remains unexplained $[5,6]$. TAC has high lipophilicity and insolubility in wa- 
ter, and is a known substrate both of the drug efflux pump, P-glycoprotein (Pgp), and metabolizing enzyme, cytochrome P450 (CYP) 3A [7]. These physicochemical properties of TAC cause large variations in oral absorption and extensive metabolism in clearance from the body. Moreover, a narrow therapeutic window necessitates therapeutic drug monitoring (TDM) to maintain efficacy of TAC [8,9] and minimize toxicity $[10,11]$.

Liver transplantation is a widely accepted treatment for end-stage hepatic disease. Despite improvements in organ preservation technology, surgical technique, and immunosuppressive strategies, postoperative biliary stricture remains a significant cause of morbidity and mortality after liver transplantation [12]. Bile duct anastomotic stricture is a common complication after orthotopic liver transplantation and has a reported prevalence of $15 \%$ $20 \%$ [13]. Previous study found that cholestasis caused by bile duct ligation induced intestinal bile deficiency and accumulation of biliary compounds in the body [14]. Therefore, decreased availability of bile salts for solubilization of lipid-soluble drugs following bile duct stricture might decrease intestinal absorption of TAC after liver transplantation. Moreover, accumulation of bile acids in liver tissue with resultant free radical production, hepatic stellate cell activation, and liver fibrosis [15-17] might decrease hepatic metabolism of TAC in liver transplantation patients. On the other hand, biliary drainage in living-related liver transplantation plays an important role in the prevention of biliary anastomotic complications and the treatment of biliary anastomotic stenosis after transplantation [18]. However, wide variations in bile flow and hepatic function in the early stage after liver transplantation with biliary drainage $[12,19]$ hinder the control of TAC concentration in TDM.

In biliary drainage at Kyoto Prefectural University of Medicine, half of the bile excreted from the drain is returned to the intestinal tract and the remaining bile is discarded. This enables collection of bile samples for determination of drug concentration and measurement of bile flow. It was previously found that TAC was mainly metabolized by the CYP 3A system in the liver and excreted in bile, whereas only a minor portion of unchanged TAC was detected in the bile $[7,20]$. Therefore, we hypothesized that measuring bile flow together with TAC and TAC metabolite levels in bile after liver transplantation with biliary drainage would be useful for the adjustment of an individual patient's dose and might be a possible strategy to improve efficacy and reduce toxicity. To investigate this hypothesis, we determined bile flow as well as TAC and TAC metabolite levels in bile from biliary drainage in the early stage after liver transplantation. This study focused on the relationship among blood TAC concentration, bile flow, and TAC metabolites in bile. In addition, the relationship between total bilirubin (T-Bil), an index of cholestasis, and blood TAC concentration was investigated in liver transplant recipients. Although several studies have performed retrospective analysis of patients who have undergone a living-related liver transplantation $[5,21]$, to our knowledge, this is the first report describing TAC and TAC metabolite levels in bile after liver transplantation with biliary drainage.

\section{Methods}

\subsection{Materials}

TAC and CyA, which was used as an internal standard in the liquid chromatography-tandem mass spectrometry (LC-MS/MS) analysis as described below, were purchased from Sigma-Aldrich Co. (Steinheim, Germany). We obtained propylene glycol from Wako Pure Chemical Industries (Osaka, Japan). All other reagents were of analytical grade and used without further purification.

\subsection{Patients}

This clinical retrospective study was conducted at the Department of Transplantation and Regenerative Surgery, Kyoto Prefectural University of Medicine, Kyoto, Japan. The study protocol was approved by the Ethics Committee of the university, and informed consent was obtained from all patients or their legal guardians (for patients aged $<18$ years). Data from the medical records of 16 male and 19 female patients, with a mean age of 38 years (range, 12 - 59 years), who had received a living-related liver transplantation at the university from 2004 through 2008 were included.

\subsection{Treatment and Study Design}

The following immunosuppressive regimen, developed by the Department of Transplantation and Regenerative Surgery for use after living-related liver transplantation, was used in the patients. Initial administration of TAC $\left(0.3 \mathrm{mg} \cdot \mathrm{kg}^{-1} \cdot \mathrm{day}^{-1}\right)$ was started orally 2 days before transplantation. On the day of transplantation, TAC $(0.1$ $\mathrm{mg} \cdot \mathrm{kg}^{-1} \cdot \mathrm{day}^{-1}$ ) was administered by continuous i.v. infusion; this was followed by oral tacrolimus $\left(0.2 \mathrm{mg} \cdot \mathrm{kg}^{-1}\right.$ $\cdot{ }^{-1 a y}{ }^{-1}$ b.i.d.). During the early stage after surgery (about 1 week), the dose of TAC was adjusted empirically on the basis of the results of frequent blood level monitoring; TAC dose was based on the trough blood level ( $\mathrm{TL}_{\mathrm{TAC}}$ ) where target $\mathrm{TL}_{\mathrm{TAC}}$ was $15 \mathrm{ng} \cdot \mathrm{mL}^{-1}$ until 3 weeks after surgery and subsequently $10 \mathrm{ng} \cdot \mathrm{mL}^{-1}$. TAC blood concentration was measured with an antibody-conjugated magnetic immunoassay (ACMIA) method by using a Dimension Xpand ${ }^{\circledR}$ system. The lower limit of detection in this method was $1.2 \mathrm{ng} \cdot \mathrm{mL}^{-1}$. Inter- and intra-assay 
reproducibility were between 5 and 10 (C.V. \%) according to results of the International Proficiency Testing Scheme. In 12 patients (6 male, 6 female) in whom bile samples could be collected, unchanged TAC and demethylated and hydroxyated metabolite concentrations in bile were measured. Bile was collected from biliary drainage twice a day after transplantation, and volume of bile was measured gravimetrically to obtain bile flow rate. The concentrations of TAC and TAC metabolites in bile were measured by an LC-MS/MS method as described below.

\subsection{Assay Procedure}

Analysis of TAC, demethyl-TAC, and hydroxy-TAC in bile was performed by LC-MS/MS [22]. The LC-MS/MS system (Applied Biosystems, Foster City, California) consisted of an LC-10AD micropump (Shimadzu Corporation, Kyoto, Japan) and an AS8020 automatic sample injector (Toso, Tokyo, Japan). Mass spectrometry was performed in the positive-ion electrospray ionization (ESI) mode. CyA was used as the internal standard. LC-MS/MS analyses were performed using a Quicksorb ODS $(2.1 \mathrm{~mm} \times 150 \mathrm{~mm}, 5 \mu \mathrm{m}$; Chemco Scientific Co. Ltd., Osaka, Japan) maintained at $60^{\circ} \mathrm{C}$ for all separations. The mobile phase consisted of $10 \mathrm{mM}$ ammonium acetate/acetonitrile $(10: 90 \mathrm{v} / \mathrm{v})$ at a flow rate of $0.2 \mathrm{~mL} / \mathrm{min}$. The ion spray voltage and temperature were set at 5000 $\mathrm{V}$ and $300^{\circ} \mathrm{C}$, respectively. The flow rates of the nebulizer gas, curtain gas, and collision gas were set at 15, 12, and $2.0 \mathrm{~L} \cdot \mathrm{mL}^{-1}$, respectively. Multiple reaction monitoring analysis was performed with transition $\mathrm{m} / \mathrm{z} 821.5 \rightarrow$ 769.7 for TAC and $\mathrm{m} / \mathrm{z} 1202.8 \rightarrow 425.2$ for CyA. Two metabolites of TAC, demethyl-TAC and hydroxy-TAC, in bile were measured with transition $\mathrm{m} / \mathrm{z} 807.5 \rightarrow 755.7$ for demethyl-TAC and $\mathrm{m} / \mathrm{z} 837.5 \rightarrow 785.7$ for hydroxyTAC. Standard samples were prepared by adding aliquots of TAC stock solutions to a drug-free matrix. The solid-phase extraction cartridges (Oasis ${ }^{\circledR}$ HLB; Waters Corp., MA, USA) were activated by $1 \mathrm{~mL}$ of acetonitrile followed by $1 \mathrm{~mL}$ of ion-exchanged water to prepare the sorbent for use. Standard and unknown samples $(100 \mu \mathrm{L})$ were added to $10 \mu \mathrm{L}$ of the internal standard (CyA, 200 $\mu \mathrm{g} / \mathrm{mL}$ in methanol) and $100 \mu \mathrm{L}$ of $2 \% \mathrm{ZnSO} 4$ in $50 \%$ methanol solution, and the tube was vortexed vigorously for $15 \mathrm{~s}$. After centrifugation for $5 \mathrm{~min}$ at $12,000 \times \mathrm{g}$, the supernatant was applied to the solid-phase extraction cartridges and subsequently washed with $2 \mathrm{~mL}$ of ionexchanged water. A $1.5 \mathrm{~mL}$ clean tube was positioned below the solid-phase extraction cartridges, and the compounds were eluted with $1 \mathrm{~mL}$ of acetonitrile. The eluate was dried with nitrogen at $37^{\circ} \mathrm{C}$. The residue was redissolved with $100 \mu \mathrm{L}$ of the mobile phase, and $50 \mu \mathrm{L}$ was injected into the LC-MS/MS system for analysis.

\subsection{Statistical Analysis}

The TAC concentration obtained after 3 days with the same dosage in each patient was considered the $\mathrm{TL}_{\mathrm{TAC}}$ and normalized according to dose $\left(\mathrm{TL}_{\mathrm{TAC}} / \mathrm{dose}\right)$. The ratio of TAC metabolite to TAC in bile (M/P) was determined by the peak area ratio obtained in LC-MS/MS analysis. Correlations between post operation days (POD) and $\mathrm{M} / \mathrm{P}$, between $\mathrm{POD}$ and $\mathrm{TL}_{\mathrm{TAC}} /$ dose, between $\mathrm{TL}_{\mathrm{TAC}} / \mathrm{dose}$ and $\mathrm{M} / \mathrm{P}$, between bile flow and T-Bil, and between T-Bile and TLTAC/dose were assessed statisticcally using Pearson's correlation coefficient test. The differences between the means were considered statisticcally significant when $p<0.05$.

\section{Results}

\subsection{Relationship among $\mathrm{M} / \mathrm{P}, \mathrm{POD}$, and TL $_{\text {TAC }} /$ Dose}

Figure 1 shows the relationships between $\mathrm{M} / \mathrm{P}$ and $\mathrm{POD}$, between $\mathrm{M} / \mathrm{P}$ and $\mathrm{TL}_{\mathrm{TAC}} /$ dose, and between $\mathrm{TL}_{\mathrm{TAC}} /$ dose and POD in living-related liver transplant patients. There was no correlation between $\mathrm{M} / \mathrm{P}$ of demethyl-TAC and POD, whereas a weak linear correlation was demonstrated between M/P of hydroxy-TAC and POD $(r=$ -0.345 ). Moreover, linear correlations were not observed between $\mathrm{M} / \mathrm{P}$ and $\mathrm{TL}_{\mathrm{TAC}} /$ dose, and between $\mathrm{TL}_{\mathrm{TAC}} /$ dose and POD.

\subsection{Relationship among Bile Flow, T-Bil, and TL $_{\text {TaC }} /$ Dose}

Figure 2 shows the relationships between bile flow and $\mathrm{T}$-Bil, and between $\mathrm{TL}_{\mathrm{TAC}}$ /dose and T-Bil, in living-related liver transplant patients. A negative linear correlation was demonstrated between bile flow and T-Bil in blood $(r=-0.495)$. Furthermore, a positive linear correlation was observed between $\mathrm{TL}_{\mathrm{TAC}}$ /dose and $\mathrm{T}$-Bil in blood $(r=0.598)$. There was no correlation between bile flow and $\mathrm{TL}_{\mathrm{TAC}} /$ dose.

\section{Discussion}

The present study aimed to investigate the effects of cholestasis on blood TAC levels in liver transplantation patients. Whereas TAC level was not correlated with bile flow in our patients with biliary drainage, a positive correlation with T-Bil suggests that T-Bil may be a useful biomarker for determining the degree of bile duct stricture and TAC dose in liver transplantation patients.

In this study, demethyl- and hydroxy-TAC were measured in bile because the main metabolites of TAC in liver-grafted patients are demethyl-TAC in blood and urine and hydroxy-TAC in bile [23]. It has been reported that the clearance of TAC is related to postoperative days 


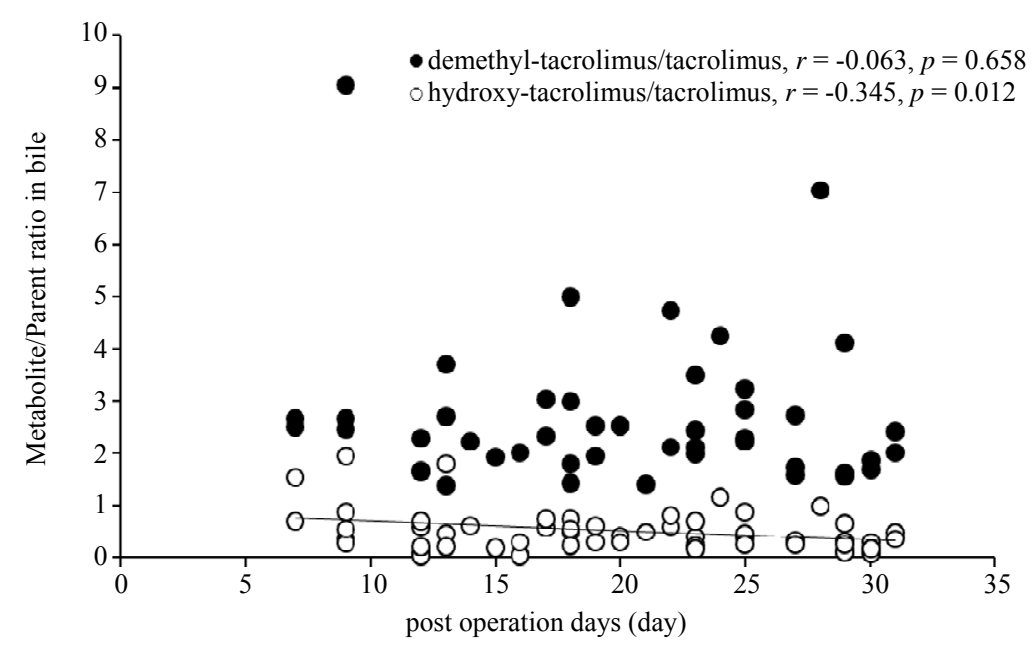

(a)

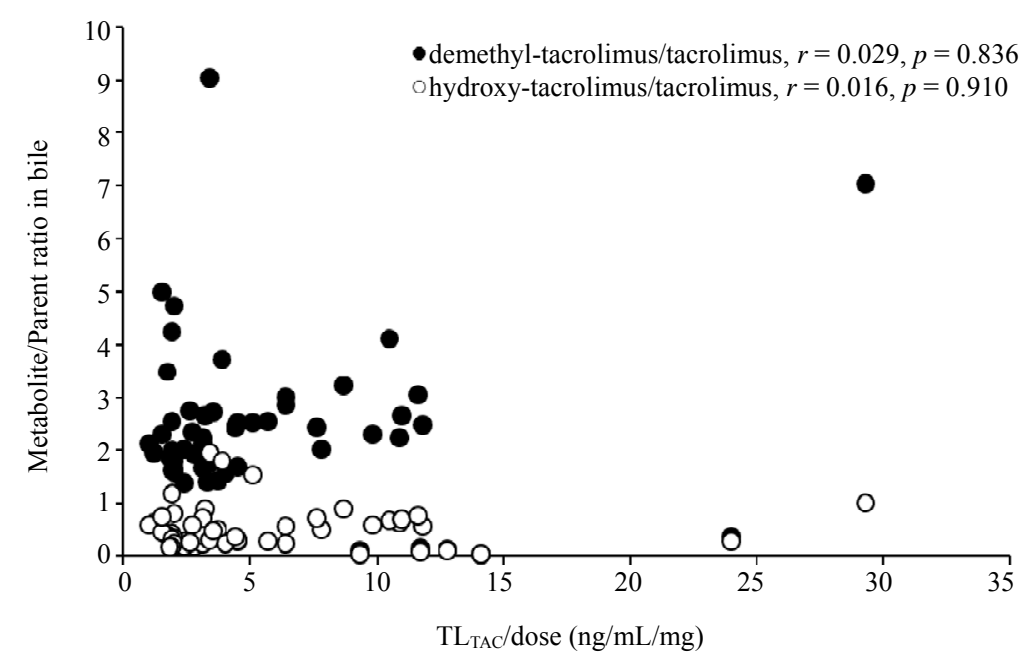

(b)

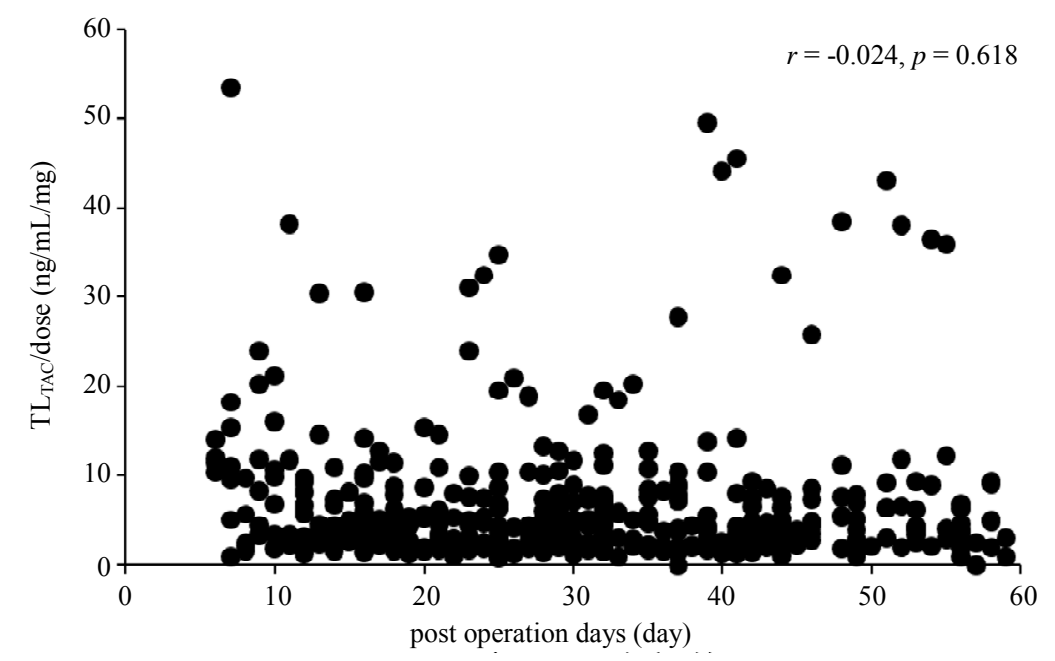

(c)

Figure 1. Relationships between the ratio of tacrolimus (TAC) metabolite to TAC (M/P) and post operation days (POD) (n = 52) (a), between M/P and TAC trough level normalized dose $\left(\mathrm{TL}_{\mathrm{TAC}} /\right.$ dose $)(n=52)(\mathrm{b})$, and between $\mathrm{TL}_{\mathrm{TAC}} /$ dose and POD $(\mathrm{n}$ $=421$ ) (c) in living-related liver transplant patients. 


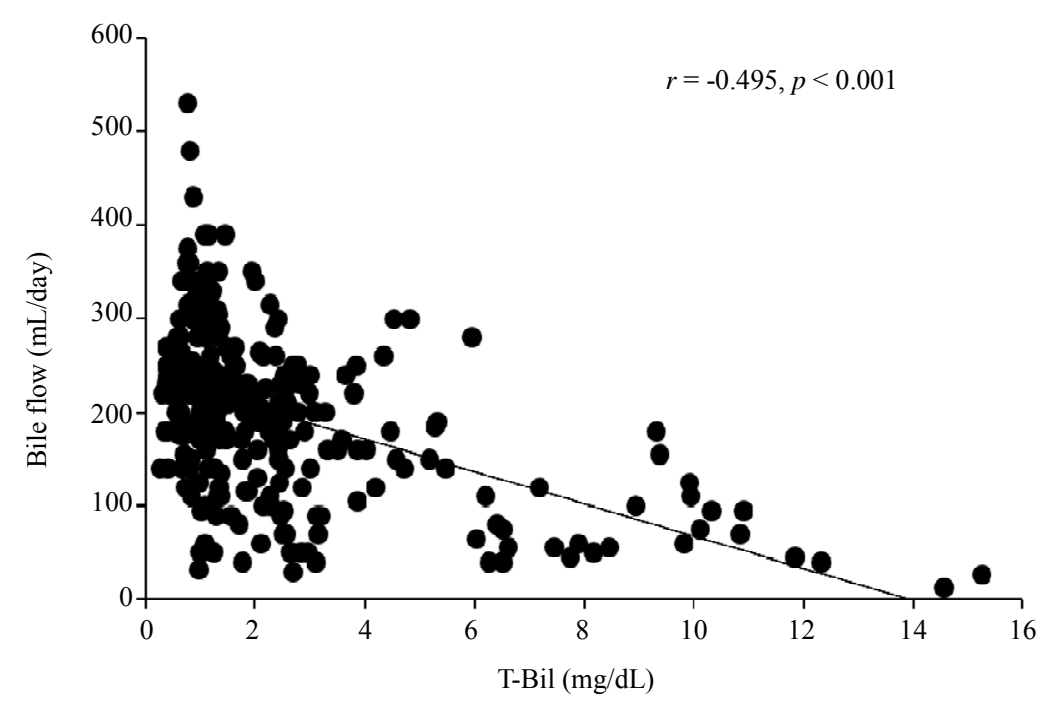

(a)

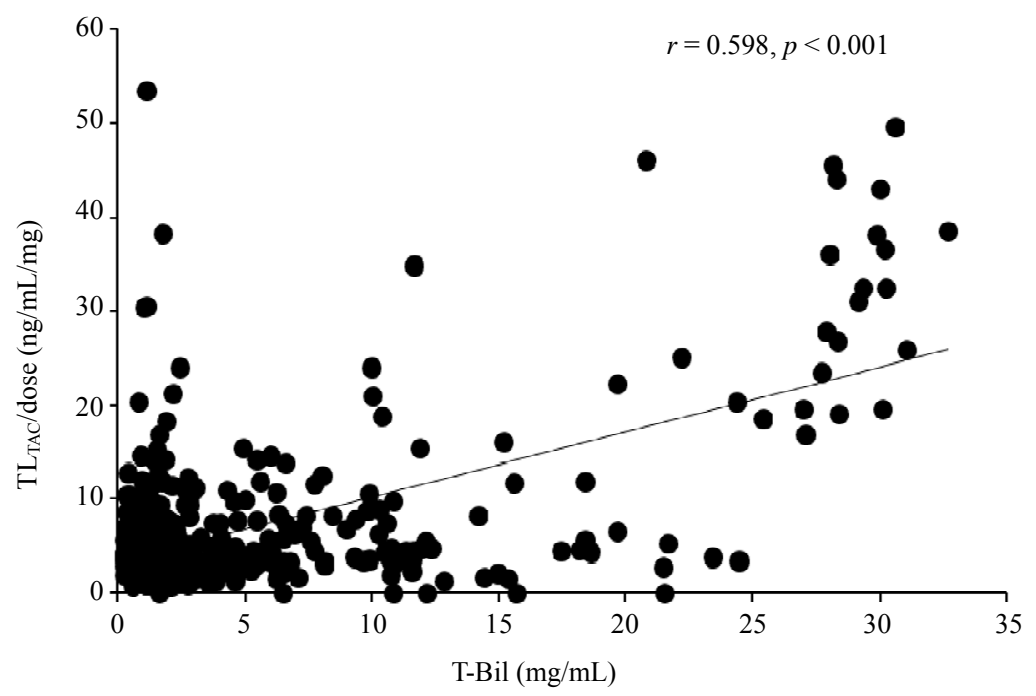

(b)

Figure 2. Relationships between bile flow and total bilirubin (T-Bil) $(n=309)(a)$ and between tacrolimus (TAC) trough level normalized dose $\left(\mathrm{TL}_{\mathrm{TAC}} /\right.$ dose $)$ and T-Bil $(\mathrm{n}=401)(\mathrm{b})$ in living-related liver transplant.

in pediatric patients receiving living-donor liver transplantation. Fukudo et al. performed population pharmacokinetic analysis with nonlinear mixed-effects modeling and reported that oral clearance of TAC increased with time in the immediate postoperative period but did not change further after postoperative day 21 [21]. Antignac et al. reported that the clearance of TAC was approximately zero immediately after surgery and then rapidly increased as a function of postoperative days to reach a plateau in adult full-liver transplant patients, which was modeled as a sigmoid relationship [24]. However, in this study, from 7 to 31 days after surgery, there was no correlation between $\mathrm{M} / \mathrm{P}$ of demethyl-TAC and POD, whereas a weak negative correlation was demonstrated between $\mathrm{M} / \mathrm{P}$ of hydroxy-TAC and POD. In addition, linear correlations were not observed between $\mathrm{M} / \mathrm{P}$ and $\mathrm{TL}_{\mathrm{TAC}} /$ dose, and between $\mathrm{TL}_{\mathrm{TAC}} /$ dose and POD. These results suggest that improvement of hepatic function and increase of TAC clearance after postoperative day 7 do not significantly contribute to hepatic TAC metabolism, bile excretion, and $\mathrm{TL}_{\mathrm{TAC}} /$ dose. It is reported that postoperative biliary stricture is a significant cause of morbidity and mortality in the early stage after liver transplantation and that cholestasis caused by postoperative biliary stricture induces intestinal bile deficiency and liver fibrosis $[12,14,17]$. These observations suggest that postoperative biliary stricture from liver transplantation with/without biliary drainage, rather than improvements 
of hepatic function with recovery from surgical damage or regeneration of the grafted liver, contributes to interand intra-patient pharmacokinetic variability of TAC.

To evaluate the contribution of postoperative biliary stricture to pharmacokinetic variability of TAC, the correlations among bile flow, $\mathrm{T}-\mathrm{Bil}$, and $\mathrm{TL}_{\mathrm{TAC}} / \mathrm{dose}$ were investigated in liver transplantation patients. A negative linear correlation was demonstrated between bile flow and T-Bil in blood, and a positive linear correlation was observed between $\mathrm{TL}_{\mathrm{TAC}}$ /dose and T-Bil in blood. It is reported that cholestasis caused by bile duct ligation induces intestinal bile deficiency and accumulation of biliary compounds in the body [14]. Moreover, we previously reported that intestinal absorption of TAC depended on the amount of bile in the intestine and that lack of bile after bile duct ligation might result in decreased TAC absorption; thus, the volume of bile in the intestine was an important factor in TAC treatment [25]. In addition, accumulation of bile acids in the liver tissue in biliary obstruction causes liver fibrosis [15-17]. These results indicate that postoperative biliary stricture is associated with inter- and intra-patient variability of $\mathrm{TL}_{\mathrm{TAC}} /$ dose level after liver transplantation, and that this can be assessed by the T-Bil level. However, in this study, there was no correlation between bile flow and $\mathrm{TL}_{\mathrm{TAC}} /$ dose. $\mathrm{A}$ possible explanation for this might be that half of the bile excreted from the drain was returned to the intestinal tract during biliary drainage, which might have strongly affected the absorption of TAC and $\mathrm{TL}_{\mathrm{TAC}} /$ dose, resulting in no significant correlation between bile flow and $\mathrm{TL}_{\mathrm{TAC}} /$ dose despite a significant linear correlation between $\mathrm{TL}_{\mathrm{TAC}} /$ dose and $\mathrm{T}$-Bil in blood. These observations suggest that T-Bil in blood might be a predictive biomarker for determining the degree of bile duct stricture and TAC dose in liver transplantation patients, and that TDM with T-Bil monitoring would be beneficial and enable individual adjustment of the dose of TAC.

This study had the following limitations: first, a relatively small sample size with study subjects recruited from a single institution, and second, the use of biliary drainage, which might have affected absorption and resultant dose of TAC because of return of bile to the intestine. These factors limit generalization of the study findings. Therefore, to evaluate the net effects of cholestasis on whole blood concentration of TAC, further investigation of a large number of subjects in a multicenter clinical trial is warranted. It would also be interesting to analyze the effect of return of bile to the intestinal tract in biliary drainage on the absorption of TAC and resultant TAC dose with a suitable statistical method.

In conclusion, the present data have several potentially important implications. First, improvement of hepatic function and increase of TAC clearance after postopera- tive day 7 do not significantly contribute to hepatic TAC metabolism, bile excretion, and $\mathrm{TL}_{\mathrm{TAC}} /$ dose. Second, postoperative biliary stricture from liver transplantation with/without biliary drainage causes inter- and intra-patient variability of $\mathrm{TL}_{\mathrm{TAC}} /$ dose level after liver transplanttation, and this can be assessed by T-Bil. Thus, T-Bil in blood might be a predictive biomarker for determining the degree of bile duct stricture and TAC dose in liver transplantation patients. Along with an appropriate dosing regimen, TDM including T-Bil would be beneficial and enable individual adjustment of the dose of TAC in liver transplantation patients.

\section{Acknowledgements}

This research received no specific grant from any funding agency in the public, commercial, or not-for-profit sectors.

\section{REFERENCES}

[1] T. Kino, H. Hatanaka, M. Hashimoto, M. Nishiyama, T. Goto, M. Okuhara, M. Kohsaka, H. Aoki and H. Imanaka, "FK-506, a Novel Immunosuppressant Isolated from a Streptomyces. I. Fermentation, Isolation, and PhysicoChemical and Biological Characteristics," The Journal of Antibiotics (Tokyo), Vol. 40, No. 9, 1987, pp. 1249-1255. http://dx.doi.org/10.7164/antibiotics.40.1249

[2] T. Kino, H. Hatanaka, S. Miyata, N. Inamura, M. Nishiyama, T. Yajima, T. Goto, M. Okuhara, M. Kohsaka, H. Aoki, et al., "FK-506, a Novel Immunosuppressant Isolated from a Streptomyces. II. Immunosuppressive Effect of FK-506 in Vitro," The Journal of Antibiotics (Tokyo), Vol. 40, No. 9, 1987, pp. 1256-1265. http://dx.doi.org/10.7164/antibiotics.40.1256

[3] G. L. Plosker and R. H. Foster, "Tacrolimus: A Further Update of Its Pharmacology and Therapeutic Use in the Management of Organ Transplantation," Drugs, Vol. 59, No. 2, 2000, pp. 323-389. http://dx.doi.org/10.2165/00003495-200059020-00021

[4] E. M. Haddad, V. C. McAlister, E. Renouf, R. Malthaner, M. S. Kjaer and L. L. Gluud, "Cyclosporin versus Tacrolimus for Liver Transplanted Patients," Cochrane Database System Review, Vol. 4, 2006, CD005161. http://dx.doi.org/10.1002/14651858.CD005161.pub2

[5] C. E. Staatz and S. E. Tett, "Clinical Pharmacokinetics and Pharmacodynamics of Tacrolimus in Solid Organ Transplantation," Clinical Pharmacokinetics, Vol. 43, No. 10, 2004, pp. 623-653.

http://dx.doi.org/10.2165/00003088-200443100-00001

[6] M. Naesens, D. R. Kuypers and M. Sarwal, "Calcineurin Inhibitor Nephrotoxicity," Clinical Journal of the American Society of Nephrology, Vol. 4, No. 2, 2009, pp. 481508. http://dx.doi.org/10.2215/CJN.04800908

[7] Tacrolimus (Prograf ${ }^{\mathbb{B}}$ ), "Drug Information," Astellas Pharma Inc., Tokyo, 2013.

[8] R. P. Kershner and W. E. Fitzsimmons, "Relationship of 
FK506 Whole Blood Concentrations and Efficacy and Toxicity after Liver and Kidney Transplantation," Transplantation, Vol. 62, No. 7, 1996, pp. 920-926. http://dx.doi.org/10.1097/00007890-199610150-00009

[9] P. McMaster, D. F. Mirza, T. Ismail, G. Vennarecci, P. Patapis and A. D. Mayer, "Therapeutic Drug Monitoring of Tacrolimus in Clinical Transplantation," Therapeutic Drug Monitoring, Vol. 17, No. 6, 1995, pp. 602-605. http://dx.doi.org/10.1097/00007691-199512000-00010

[10] G. W. Boswell, I. Bekersky, J. Fay, J. Wingard, J. Antin, D. Weisdorf, R. Maher, W. Fitzsimmons and R. Nash, "Tacrolimus Pharmacokinetics in BMT Patients," Bone Marrow Transplant, Vol. 21, No. 1, 1998, pp. 23-28. http://dx.doi.org/10.1038/sj.bmt.1701054

[11] W. J. Jusko, W. Piekoszewski, G. B. Klintmalm, M. S. Shaefer, M. F. Hebert, A. A. Piergies, C. C. Lee, P. Schechter and Q. A. Mekki, "Pharmacokinetics of Tacrolimus in Liver Transplant Patients," Clinical Pharmacology \& Therapeutics, Vol. 57, 1995, pp. 281-290. http://dx.doi.org/10.1016/0009-9236(95)90153-1

[12] R. J. Stratta, R. P. Wood, A. N. Langnas, R. R. Hollins, K. J. Bruder, J. P. Donovan, D. A. Burnett, R. P. Lieberman, G. B. Lund, T. J. Pillen, et al., "Diagnosis and Treatment of Biliary Tract Complications after Orthotopic Liver Transplantation," Surgery, Vol. 106, 1989, pp. 675-683.

[13] J. W. Ostroff, "Post-Transplant Biliary Problems," Gastrointestinal Endoscopy Clinics of North America, Vol. 11, 2001, pp. 163-183.

[14] D. M. Minich, R. Havinga, F. Stellaard, R. J. Vonk, F. Kuipers and H. J. Verkade, "Intestinal Absorption and Postabsorptive Metabolism of Linoleic Acid in Rats with Short-Term Bile Duct Ligation," American Journal of Physiology-Gastrointestinal and Liver Physiology, Vol. 279, No. 6, 2000, pp. G1242-G1248.

[15] R. J. Sokol, M. Devereaux, R. Khandwala and K. O'Brien, "Evidence for Involvement of Oxygen Free Radicals in Bile Acid Toxicity to Isolated Rat Hepatocytes," Hepatology, Vol. 17, No. 5, 1993, pp. 869-881. http://dx.doi.org/10.1016/0270-9139(93)90164-I

[16] G. Poli, "Pathogenesis of Liver Fibrosis: Role of Oxidative Stress," Molecular Aspects of Medicine, Vol. 21, No. 3, 2000, pp. 49-98. http://dx.doi.org/10.1016/S0098-2997(00)00004-2

[17] G. Tahan, H. Akin, F. Aydogan, S. S. Ramadan, O. Yapicier, O. Tarcin, H. Uzun, V. Tahan and K. Zengin, "Melatonin Ameliorates Liver Fibrosis Induced by BileDuct Ligation in Rats," Canadian Journal of Surgery, Vol. 53, 2010, pp. 313-318.
[18] S. Eguchi, M. Takatsuki, A. Soyama, M. Hidaka, I. Muraoka and T. Kanematsu, "Use of Stepwise versus Straightforward Clamping of Biliary Drainage Tubes after Living-Donor Liver Transplantation: A Prospective, Randomized Trial," Journal of Hepato-Biliary-Pancreatic Surgery, Vol. 19, No. 4, 2012, pp. 379-381.

http://dx.doi.org/10.1007/s00534-011-0424-3

[19] M. L. Shiffman, R. L. Carithers Jr., M. P. Posner and E. W. Moore, "Recovery of Bile Secretion Following Orthotopic Liver Transplantation," Journal of Hepatology, Vol. 12, No. 3, 1991, pp. 351-361. http://dx.doi.org/10.1016/0168-8278(91)90839-4

[20] R. Venkataramanan, A. Jain, V. S. Warty, K. Abu-Elmagd, M. Alessiani, J. Lever, A. Krajak, J. Flowers, S. Mehta, S. Zuckerman, et al., "Pharmacokinetics of FK 506 in Transplant Patients," Transplant Proceedings, Vol. 23, No. 6, 1991, pp. 2736-2740.

[21] M. Fukudo, I. Yano, S. Masuda, M. Goto, M. Uesugi, T. Katsura, Y. Ogura, F. Oike, Y. Takada, H. Egawa, S. Uemoto and K. Inui, "Population Pharmacokinetic and Pharmacogenomic Analysis of Tacrolimus in Pediatric Living-Donor Liver Transplant Recipients," Clinical Pharmacology \& Therapeutics, Vol. 80, 2006, pp. 331345. http://dx.doi.org/10.1016/j.clpt.2006.06.008

[22] R. A. Koster, E. C. Dijkers and D. R. Uges, "Robust, HighThroughput LC-MS/MS Method for Therapeutic Drug Monitoring of Cyclosporine, Tacrolimus, Everolimus, and Sirolimus in Whole Blood," Therapeutic Drug Monitoring, Vol. 31, No. 1, 2009, pp. 116-125. http://dx.doi.org/10.1097/FTD.0b013e318192304c

[23] U. Christians, F. Braun, N. Kosian, M. Schmidt, H. M. Schiebel, L. Ernst, C. Kruse, M. Winkler, I. Holze, A. Linck, et al., "High Performance Liquid Chromatography/ Mass Spectrometry of FK 506 and Its Metabolites in Blood, Bile, and Urine of Liver Grafted Patients," Transplant Proceedings, Vol. 23, 1991, pp. 2741-2744.

[24] M. Antignac, J. S. Hulot, E. Boleslawski, L. Hannoun, Y. Touitou, R. Farinotti, P. Lechat and S. Urien, "Population Pharmacokinetics of Tacrolimus in Full Liver Transplant Patients: Modelling of the Post-Operative Clearance," European Journal of Clinical Pharmacology, Vol. 61, No. 5-6, 2005, pp. 409-416. http://dx.doi.org/10.1007/s00228-005-0933-6

[25] S. Kobuchi, K. Fukushima, Y. Maeda, T. Kokuhu, H. Ushigome, N. Yoshimura, N. Sugioka and K. Takada, "Effects of Bile Duct Stricture on the Pharmacokinetics of the Immunosuppressant Tacrolimus in Rats," Interactive Medicinal Chemistry, in press. 


\section{Abbreviations}

ACMIA: Antibody-conjugated magnetic immunoassay; CyA: Cyclosporine;

CYP: Cytochrome P450;

ESI: Electrospray ionization;

LC-MS/MS: The liquid chromatography-tandem mass spectrometry;

$\mathrm{M} / \mathrm{P}$ : The ratio of TAC metabolite to TAC in bile;
Pgp: P-glycoprotein;

POD: Post operation days;

TAC: Tacrolimus;

T-Bil: Total bilirubin;

TDM: Therapeutic drug monitoring;

$\mathrm{TL}_{\mathrm{TAC}}$ : Trough blood level;

$\mathrm{TL}_{\mathrm{TAC}} /$ dose: $\mathrm{TAC}$ trough level normalized dose. 